\title{
Digital speech recording and playback system using a multiprocessor architecture
}

\author{
GEORGE J. BOGGS, RICHARD ALLEY, and MARK GUZDIAL \\ GTE Laboratories, Incorporated, Waltham, Massachusetts
}

This paper describes a digital speech recording system capable of digitizing, storing, and playing back speech signals of arbitrary duration. The maximum duration for continuous speech processing is limited only by disk storage capacity. The system is based on a multiprocessor architecture, with a main processor responsible for file processing and outboard processor $\mathrm{I} / \mathrm{O}$ control. As implemented, the main processor is a DEC LSI-11/23 microcomputer with $1 \mathrm{MB}$ of main memory and a large-capacity Winchester disk. The outboard processor is an Intel 8085 with $2 \mathrm{~K}$ local memory for speech buffering. Digitization is accomplished using logarithmic PCM at 8-kHz sampling frequency. Interprocessor communication is achieved with a 16-bit parallel data bus, and the necessary electrical connections and handshaking signals are discussed in detail. The local software modules are described, with attention given to timing and communications protocol considerations.

In speech and linguistic research, it is frequently desirable to digitally record and/or play back natural human speech. To do this, sampling rates for digital conversions must be as precise as possible to avoid distortion. In addition, it is often desirable to store the encoded speech on a peripheral mass-storage device like a Winchestertype disk. If long speech passages are processed, memory constraints may require that digital encoding/decoding operations be interleaved with disk read/write operations. Bandwidth requirements for interleaved speech I/O and disk operations can exceed the bandwidth capacity of many commercially available microprocessors.

This paper describes a speech $\mathrm{I} / \mathrm{O}$ system based on a multiprocessor architecture. Our laboratory uses a Digital Equipment Corporation LSI-11/23 microprocessor with the RSX-11/M operating system, and our goal was to provide speech $I / O$ with constant sampling rate and disk-access capability. The LSI-11/23 microcomputer has insufficient bandwidth to perform this task. Therefore, we designed a system that can encode/decode a speech sample of arbitrary duration to or from a Winchester disk. The only constraint on the duration of the speech sample is the amount of available disk space.

\section{FUNCTIONAL ARCHITECTURE}

A block diagram of the system is shown in Figure 1. Functionally, the system consists of two independently running microprocessors for analog sampling and disk storage. A high-speed parallel data bus is used for interprocessor communication. Our system uses an outboard microprocessor for either analog-to-digital (ADC) or

The authors are affiliated with the Telecommunications Research Laboratory, Human Factors Department, GTE Laboratories, Inc., 40 Sylvan Road, Waltham, MA 02254. digital-to-analog (DAC) sampling. The outboard processor switches between recording (ADC) and playback (DAC) modes in response to signals from the main processor.

When an experiment calls for speech input and storage, the main processor opens a disk file and allocates memory for a speech buffer. The outboard processor has, on board, dedicated memory space for speech buffering. An input operation is initiated by the main processor, and the outboard processor (with local clocking) is switched into ADC mode. The outboard processor samples and buffers the speech locally. When the main processor is available for a disk write, the outboard processor is notified, and data transfer via the parallel data bus is performed. When the required number of speech samples have been digitized, the main processor effectively terminates the input operation (by halting data input-the 8085 microprocessor continues to sample, however).

Speech output is a symmetrical operation. The main processor switches the outboard processor into DAC mode, opens the disk file, and reads a buffer full of speech samples. Data transfer is initiated to the outboard processor's speech buffer for DAC operation. When the last sample has been converted by the outboard processor, the main processor effectively terminates the DAC operation (again, by terminating output of data).

\section{HARDWARE}

The main processor system, in our implementation, contains a Digital Equipment Corporation (DEC) LSI11/23 (Model KEV-11AA) microprocessor, $1 \mathrm{MB}$ of memory, and a memory-management unit. For mass storage, the system employs a Control Data Corporation (CDC) 161-MB Winchester-type disk emulated as two DEC RM03 disk drives. The disk controller board is an 


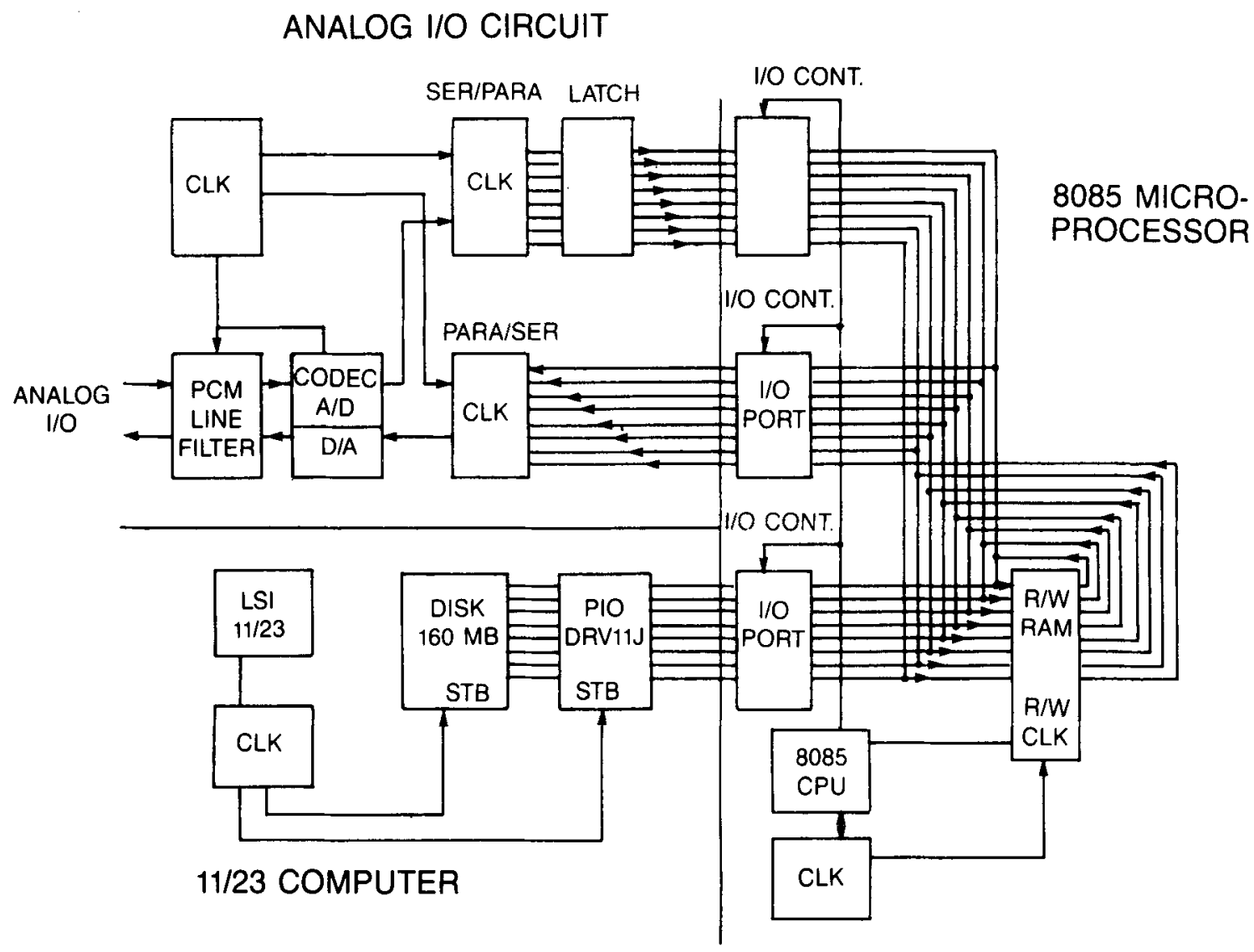

Figure 1. Functional block diagram of speech $\mathrm{I} / \mathrm{O}$ system with multiprocessor architecture.

Emulex SC03/B1. An important point about this particular disk controller board is that the board is intelligent enough to allow asynchronous data transfers (relative to the LSI-11/23 CPU). Therefore, the CPU need only initiate a disk transfer. The disk controller then completes the transfer without further intervention. Asynchronous file-transfer capability conserves system bandwidth for time-critical interprocessor communications.

The LSI-11/23 microprocessor is interfaced to a DEC DRV11-J parallel I/O board. The DRV11-J has four independently programmable ports, and each port is 16 bits wide. For our application, one port (port C) is used for data $\mathrm{I} / \mathrm{O}$. We wanted to avoid interrupt-driven data transfer, since interrupt processing with the operating system requires complex systems programming. Therefore, port D of the DRV11-J was used as a register for reading handshaking signals via status bits.

The outboard processor is an Intel 8085 microprocessor board with 2,512 bytes of random-access memory (RAM): 512 bytes on two $8155 \mathrm{RAM} /$ port drivers and 2K of expansion RAM (Intel 8155). The speech buffer is contained in the $2 \mathrm{~K}$ expansion RAM; the remainder is used as a monitor scratchpad or is unused. The $\mathbf{8 0 8 5}$ microprocessor uses an on-board system clock and coderdecoder (CODEC) hardware for speech digitization. Before and after digitization, the analog speech waveforms are filtered through cascaded Krohn-Hite 3322 tunable bandpass filters (Butterworth characteristic with 48-dB/octave rolloff). The frequency response of the system is ultimately limited by the Nyquist frequency. To prevent low-frequency foldover (aliasing), and to be consistent with telephony practice, the bandpass filters in our system are set for the $300-3400-\mathrm{Hz}$ band. The $3400-\mathrm{Hz}$ upper cutoff frequency is significantly lower than the Nyquist frequency $(4.0 \mathrm{kHz})$, thus precluding perceptually significant aliasing noise.

The functions of digital recording and playback were accomplished by using $\mu$-law pulse code modulation $(\mu \mathrm{PCM}) \mathrm{DAC}$ and ADC hardware. The singular advantage of $\mu \mathrm{PCM}$ coding for speech is that the quantization noise power is proportional to speech power, thus ensuring a constant signal-to-noise ratio. With linear PCM, quantization noise power is constant, and signal-to-noise ratios are lower for low-power speech segments (Witten, 1982). Since our human-factors research involves experiments using telephone-quality speech, and telephone channels are bandlimited between 300 and $3400 \mathrm{~Hz}$, a sampling rate of $8 \mathrm{kHz}$ was adopted. The DAC and ADC were both 8 bits wide, again consistent with standard telephony practice.

The main challenge in implementing digital recording and playback was the interface design for the outboard processor and the DRV11-J I/O board. This required a combination of hardware and software design for the 8085 
microprocessor. The design of the interface involved two main points: handshaking and electrical connections. Handshaking and electrical connections establish the communications protocol for physically transferring data between the two processors.

The DRV11-J requires that a particular sequence of handshaking signals be exchanged when data are transferred to and from an attached device. Figures 2 and 3 display the sequence of handshaking signals, USER RPLY, DRV11-J RPLY, and USER RDY, that occur for input and output. In addition, the DRV11-J must be able to switch the 8085/CODEC between ADC and DAC modes. This is accomplished with the DRV11-J RDY output signal.

The reception and generation of handshaking signals by the 8085 could have been realized in software. Instead, we selected hardware handshaking for the following reasons. If one 8085 port was used to receive incoming signals, another port could have been used to send the return signals. The 8085 microprocessor would then have polled the input port for a handshake signal and, upon its receipt, sent the appropriate reply. This method, however, is very slow and inefficient; much time is lost polling and sending signals. Also, there are times when the 8085 services interrupts from the CODEC/clock circuit and is unable to perform the handshaking. Since the DRV11-J has a definite "window" during which actual data transfer must occur, data can be lost when the 8085 is performing other services.

To expedite $\mathrm{I} / \mathrm{O}$ handling for the 8085 , the Intel 8155 RAM and I/O port driver, programmed through a command register, performs data transfers with hardware handshaking. Using this method, the 8085 is easily synchronized with the DRV11-J while low processor overhead is maintained.

In our system, all data transfers are accomplished in hardware. This ensures that the necessary average data-

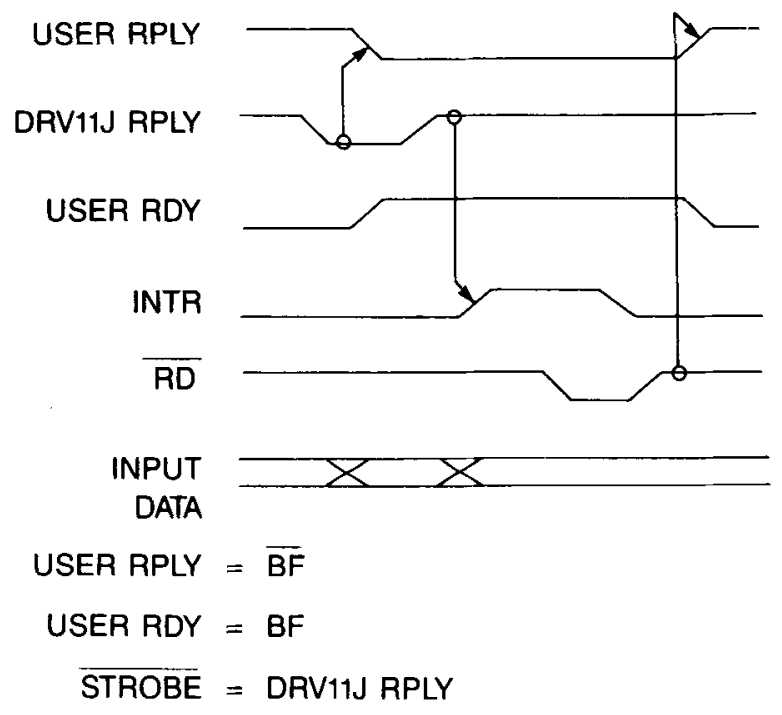

Figure 2. Output mode (ADC) handshake timing.

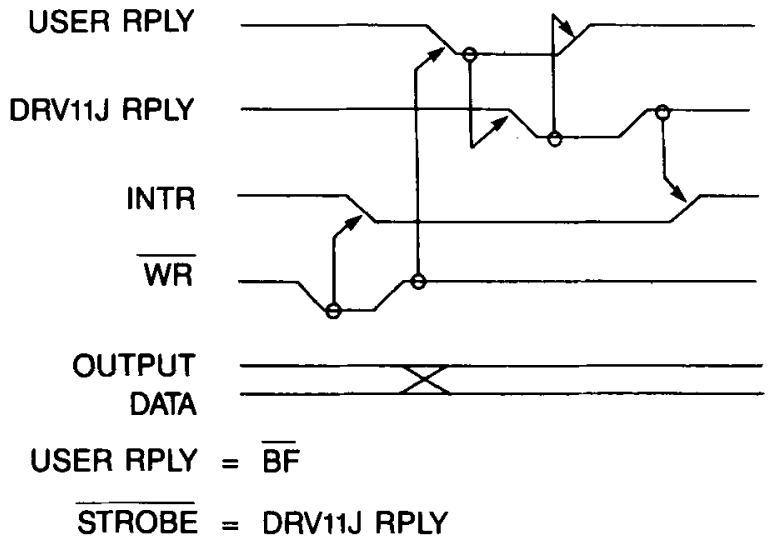

Figure 3. Input mode (DAC) handshake timing.

transfer rate of $8 \mathrm{kHz}$ between the two processors can be maintained while the processor is busy with buffer housekeeping. The 8085 merely polls the INTR bit of the 8155 status register and performs an INPUT or OUTPUT to a data port. The performance of an $\mathrm{I} / \mathrm{O}$ operation by the 8085 , as signified by the arrival of a RD or WR signal at the 8155 , causes the 8155 to begin the next data transfer. No further processor intervention is required, and the 8085 may be devoted to updating the buffer.

The physical connection of the DRV11-J parallel interface to the 8085 microprocessor required three ports, as shown in Figure 4. Port 23H monitors DRV11-J RDY. When asserted, DRV11-J RDY forces the 8085/CODEC to enter DAC mode. A low DRV11-J RDY signal initiates ADC mode. Port $2 \mathrm{BH}$ sends and receives the handshaking signals. Here BF (buffer full) at pin 1 is inverted to produce USER RPLY. USER RPLY is generated by a double inversion of BF rather than a direct connection to pin 1. The double inversion was done because the $\mathbf{8 1 5 5}$ RAM port driver is unable to sink enough current to force the handshaking lines low through a direct connection. Data are sent and received through port $2 \mathrm{AH}$.

During testing, high-frequency noise was detected in the analog output of the CODEC. The problem was finally resolved by separating the ground connections for the analog (e.g., CODEC and associated hardware) sections of the board from the digital sections. Since this is an easy problem to overlook, and very difficult to diagnose, it is worth particular attention by the prospective builder.

\section{SOFTWARE}

\section{Outboard Processor}

During a disk access by the LSI-11/23, no data can be transmitted or received by the DRV11-J; to ensure an unbroken stream of data to and from the CODEC, all data transfers must be buffered by the 8085 . Since the order of data must be preserved, data must leave the buffer in the same order they entered; the buffer must be first-in first-out (FIFO). The buffering must also operate in two different modes, ADC and DAC. 

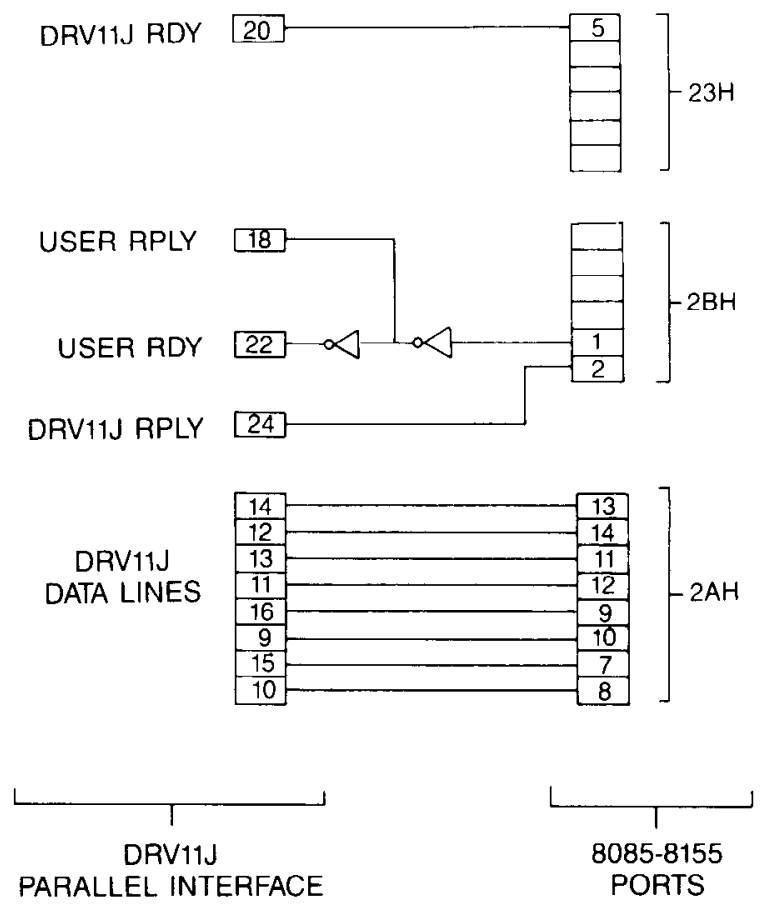

Figure 4. Pin connections for parallel data bus $\mathrm{I} / \mathrm{O}$ and handshaking.

In DAC mode, the DRV11-J is sending data to the buffer while the CODEC is reading data from the buffer. In this mode, DRV11-J must not overwrite data not yet read by the CODEC-any data overwritten will be lost. The CODEC must also read each byte of data once and only once; reading data more than once introduces noise. When the CODEC has read all available data, silence data (FF in hexadecimal) should be sent to the CODEC. If silence data is not sent after speech playback is complete, the DAC will treat the data simply as more output. Noise will be sent to the listener unless the headphones (or speaker) are switched out of the circuit.

In the ADC mode, DRV11-J reads from the buffer while the CODEC writes to the buffer. Here, DRV11-J must read the data only once, or noise will be recorded. Also, the CODEC must not overwrite data not yet read by DRV11-J. Since the CODEC will always be writing data to the buffer, even if the buffer is not being read, the CODEC eventually fills a finite-sized buffer. If the CODEC then stops, when DRV11-J next reads the buffer it receives data from speech at some indefinite time in the past. This defeats precise control over speech input.

The FIFO buffer software uses a single buffer accessed simultaneously by both the DRV11-J and the CODEC. Both the CODEC and DRV11-J have pointers indicating the next buffer position available for access. The rules for the operation of the buffer described previously are implemented by making comparisons of the relative positions of the buffer pointers. This method ensures precise control over the time period when the DRV11-J transfers data and prevents data loss/duplication.

Once the software and handshaking for the 8085 were debugged, one critical test of the system remained. Although the instantaneous rate of data exchange with DRV11-J may vary widely, the average rate must equal the $8-\mathrm{kHz}$ sampling rate of the CODEC. The limitation on the average rate of transfer is the longest time between any two data transfers. The longest time occurs when a disk access is performed. To prevent a loss of data, the longest time between data transfers must be less than the time required to empty or fill the buffer. In the best-case situation for DAC, data transfer with the DRV11-J might halt when the buffer is full. If the DRV11-J does not resume data input before the CODEC has read all the buffer, a silent gap will appear in the output speech. This is obviously unacceptable.

An average data-transfer rate of $8 \mathrm{kHz}$ can be achieved by using a $2 \mathrm{~K}$ buffer. Smaller buffers may not provide enough time for RSX-11M to do the necessary file operations. A $2 \mathrm{~K}$ buffer provides a maximum of $250 \mathrm{msec}$ for disk access in the worst-case situation. The main processor device handlers were also written to input and output only in $2 \mathrm{~K}$ blocks. This guarantees a sufficient amount of time for disk access.

After error-free communication had been established between the 8085/CODEC board and the DRV11-J parallel interface, some final bugs were discovered. We found persistent high-frequency noise in the analog output of the CODEC. Initially, we thought this noise was being produced by the supportive hardware for the CODEC. After it was determined that the hardware was not the source, the 8085 software was examined. We discovered that each byte of data was appearing twice in the buffer in the DAC mode. The duplication of data was tracked back to an idiosyncracy of the main processor and the DRV11-J. The data register on the DRV11-J is 16 bits wide. The speech data (on our system) are only 8 bits wide. When we attempted to write a byte of data into the DRV11-J data register, the $11 / 23$ processor always aligned its data fetch to the nearest even address to create a 16-bit word. If the data byte occupied an odd address, the immediately preceding (even address) byte was also fetched and deposited in the DRV11-J data regiser. The main processor software was actually the source of the problem. Once diagnosed, the problem was trivial to fix, but diagnosing the problem was difficult. Usually, the $11 / 23$ will issue a trap error if address boundaries are violated, but in this case no trap error was issued.

Finally, the operation of the $8085 /$ CODEC can be simplified by eliminating the need for keyboard input to execute the buffer program. This is accomplished by replacing the ROM containing the 8085 monitor with an EPROM containing a modified version. Changing one jump instruction address in the monitor causes the $\mathbf{8 0 8 5}$ buffer program to begin execution on startup or reset of the 8085.

\section{Main Processor}

The major difficulty in the development of both a file handler and a device handler was the operating system on the main processor, RSX-11M. RSX-11M is a mul- 
tiuser, multitasking operating system with memorymanagement support. It is an exceptionally powerful realtime operating system for a microcomputer of this size. Because of the speed considerations inherent in real-time operations, all programming must be done in MACRO11 (assembly language).

For main processor software design, the first decision that must be made is the choice of file-access methods. RSX-11M supports a number of different file-access modes. One of these modes, RMS-11, is a fairly easy-touse, business-oriented file-access system. RMS-11 has very slow file operations because of the overhead involved in translating the easy-to-use, logical file framework to the physical level of the disk. The directive level of file access represents the opposite end of the difficulty spectrum. The user simply calls the operating system to perform physical disk accesses at supplied disk and memory addresses. RMS-11 file control procedures are easy to implement and maintain, but are not appropriate for the speed and real-time requirements of the application. Directive-level software is very fast, but is also extremely complex and difficult to maintain (ease of maintenance is usually necessary in a research environment, since software requirements often change as experiments change). FCS-11 (File Control System-11) block I/O is intermediate in difficulty and speed. FCS-11 features physical disk block access (for speed and low system overhead) with regard to file boundaries (for ease of use). Most importantly, FCS-11 allows a calling program to continue executing while the disk access is executed by the intelligent controller. Asynchronous data transfer provides additional speed advantages. However, software overhead is required to prepare the disk controller, and the time consumed by this operation can present a formidable challenge to the programmer.

During system development, one might be tempted to use the multitasking capability of RSX-11M to improve the speed of the system and the amount of memory that the entire process might access for disk buffering. Expanding the buffer can be particularly attractive, since a single task can occupy only $32 \mathrm{~K}$ words. Separate tasks can be loaded for file and device handling, effectively increasing limited memory access. The two tasks can share information, so that minimum time is wasted in processing the file access.

There are two problems with this scheme. First, RSX$11 \mathrm{M}$ permits two concurrently active tasks to communicate only through system-defined synchronization signals ("event flags" in the RSX-11M terminology, which are simply binary semaphores). This is not sufficient to assure rapid data transfer. Operating system overhead is too great to permit time-critical operations. Second, for this system to operate correctly, the line time clock must be disabled. This means that the system scheduler is a prioritized, interrupt-driven algorithm rather than a round- robin algorithm. Therefore, the operating system begins execution of a new task (other than the one that is currently executing) only after sensing I/O interrupts (referred to as "significant events" in RSX terminology) and not upon any sort of timing operation. Tasks that do not interrupt the operating system for $\mathrm{I} / \mathrm{O}$ (such as device handlers that are resident in the operating system) effectively lock out all other tasks on the system. The CPUintensive task can relinquish the processor by executing a "declare significant event" system directive (a call into the operating system for a specific operating system function to be performed), but scheduling this correctly during a real-time process is probably more difficult than beneficial.

The final decision that must be made concerns the design of the DRV11-J I/O software. In general, the implementation of a device handler can be made much simpler by not installing a driver directly into RSX-11M. Although installing a driver into the operating system $\mathrm{I} / \mathrm{O}$ structure makes a number of RSX-11M I/O control features available to the programmer, it also increases software overhead.

The alternative to an operating system resident driver is the creation of an address partition in high memory. Since all I/O on the LSI-11/23 is memory mapped to high memory, a common address partition permits the writing of very simple software modules to handle $I / O$. This precludes, of course, interrupt-driven I/O. Using an address partition, the program simply polls the status bits of the DRV11-J control register for handshaking signals from the 8085. In this system, the critical status bit is USR RPLY. The data can also be read simply by reading or writing to an address in the partition.

\section{SUMMARY}

The system, as described, has been built and tested. It can be used to record/play back human speech segments of arbitary duration (the duration is limited only by massstorage considerations). In research applications in which lengthy segments of speech are needed, this system provides an attractive alternative to analog tape recording, albeit at somewhat greater cost. However, digital voice storage does not suffer from the noise problems that accrue over time with conventional analog recordings, and permits easy digital manipulation of speech signals (random presentation, editing, filtering, analysis, etc.).

\section{REFERENCE}

Witten, I. H. (1982). Principles of computer speech. New York: Academic Press.

(Manuscript received March 21, 1984; revision accepted for publication July 20,1984 .) 